\title{
Theta-Alpha Oscillations Bind the Hippocampus, Prefrontal Cortex, and Striatum during Recollection: Evidence from Simultaneous EEG-fMRI
}

\author{
Nora A. Herweg, ${ }^{1 *}$ Thore Apitz, ${ }^{1 *}$ Q Gregor Leicht, ${ }^{2}$ Christioph Mulert, ${ }^{2}$ Lluís Fuentemilla, ${ }^{3,4}$ and Nico Bunzeck ${ }^{1,5}$ \\ ${ }^{1}$ Department of Systems Neuroscience and ${ }^{2}$ Psychiatry Neuroimaging Branch, Department of Psychiatry and Psychotherapy, University Medical Center \\ Hamburg-Eppendorf, 20246 Hamburg, Germany, ${ }^{3}$ Cognition and Brain Plasticity Unit, Institute of Biomedical Research of Bellvitge, 08907 L'Hospitalet, \\ Barcelona, Spain, ${ }^{4}$ Department of Basic Psychology, University of Barcelona, 08035 Barcelona, Spain, and ${ }^{5}$ Department of Psychology, University of Lübeck, \\ 23562 Lübeck, Germany
}

Recollection of contextual information represents the core of human recognition memory. It has been associated with theta ( $4-8 \mathrm{~Hz}$ ) power in electrophysiological recordings and, independently, with BOLD effects in a network including the hippocampus and frontal cortex. Although the notion of the hippocampus coordinating neocortical activity by synchronization in the theta range is common among theoretical models of recollection, direct evidence supporting this hypothesis is scarce. To address this apparent gap in our understanding of memory processes, we combined EEG and fMRI during a remember/know recognition task. We can show that recollection-specific theta-alpha $(4-13 \mathrm{~Hz})$ effects are correlated with increases in hippocampal connectivity with the PFC and, importantly, the striatum, areas that have been linked repeatedly to retrieval success. Together, our results provide compelling evidence that low-frequency oscillations in the theta and alpha range provide a mechanism to functionally bind the hippocampus, PFC, and striatum during successful recollection.

Key words: fMRI/EEG; hippocampus; recollection; striatum; theta-alpha

\section{Significance Statement}

Low-frequency oscillations are supposed to drive the binding of information across a large-scale network centered on the hippocampus, which supports mnemonic functions. The electrophysiological means to investigate this phenomenon in humans (EEG/MEG), however, are inherently limited by their spatial resolution and therefore do not allow a precise localization of the brain regions involved. By combining EEG with BOLD-derived estimates of hippocampal connectivity during recognition, we can identify the striatum and specific areas in the medial and lateral PFC as part of a circuit linked to low-frequency oscillations (4-13 $\mathrm{Hz}$ ) that promotes hippocampus-dependent context retrieval. Therefore, the current study closes an apparent gap in our understanding of the network dynamics of memory retrieval.

\section{Introduction}

The medial temporal lobe (MTL), and especially the hippocampus, constitutes a central hub in a complex network supporting

\footnotetext{
Received Sept. 30, 2015; revised Jan. 15, 2016; accepted Feb. 12, 2016.

Author contributions: N.A.H., T.A., G.L., C.M., L.F., and N.B. designed research; T.A. performed research; N.A.H. analyzed data; N.A.H., L.F., and N.B. wrote the paper.

This work was supported by the German Research Foundation (Deutsche Forschungsgemeinschaft Grant BU 2670/2-1 to N.B.), the Faculty of Medicine of the University Medical (enter Hamburg-Eppendorf (T.A.), and the G.A. Lienert Foundation (N.A.H.). We thank Simon Pfeifer and Jonas Rauh for assistance in data acquisition and Tobias Sommer and Pablo Ripollés for support with data analysis.

The authors declare no competing financial interests.

*N.A.H. and T.A. contributed equally to this work.

Correspondence should be addressed to either Nora A. Herweg, Martinistrasse 52, 20246 Hamburg, Germany, E-mail:n.herweg@uke.de; or Nico Bunzeck, Department of Psychology, University of Lübeck, Ratzeburger Allee 160, 23562 Lübeck, Germany, E-mail: nico.bunzeck@uni-luebeck.de.
}

episodic memory functions (Battaglia et al., 2011). It has repeatedly been implicated in forming and retrieving associations (Mayes et al., 2007), which are the fundamental basis underlying recollection of contextual information (Yonelinas, 2002). An influential model (Norman and O'Reilly, 2003) describes how presentation of an item during recognition may lead to reinstatement of the pattern of cortical activity present during encoding (i.e., the item together with its contextual associations) via pattern completion in the hippocampus. While reinstatement engages primary and higher sensory cortices, the hippocampus also interacts with areas implicated in cognitive control to facilitate 
adaptive retrieval. In this regard, the PFC may direct a strategic search for stored representations and maintain relevant information in working memory (Simons and Spiers, 2003).

More recently, attention has been drawn to striatal functions complementing those of the PFC: the striatum signals retrieval success by increased activity for successfully recognized items in contrast to correctly rejected new items. Therefore, it may provide a gating mechanism for stored information and accomplish evaluation and reinforcement of retrieval strategies (Scimeca and Badre, 2012). Importantly, its functional properties with regard to recollection and familiarity and its physiological connectivity with the PFC and hippocampus during recognition remain unclear.

Long-rage communication and coordination of segregated brain regions is crucial for successful retrieval. A key neurophysiological mechanism assumed to fulfill this function is oscillatory activity in the theta frequency range (Klimesch et al., 2010; Colgin, 2013). Theta oscillations might facilitate complementary functional processes on two levels: they bind multiple features or items to form one coherent representation and they coordinate different brain regions engaged in different subprocesses of complex working memory functions by long-range synchronization (Sauseng et al., 2010). Compared with mere stimulus familiarity, recollection of contextual information should require higher coordination on a representational and regional level and, therefore, has been associated with increased theta power (Guderian and Düzel, 2005) and interregional phase synchronization (Fuentemilla et al., 2014).

Together, these lines of research can be reconciled in a common framework implicating theta oscillations in coordinating hippocampal interactions with segregated brain areas during retrieval, but direct evidence remains scarce. Therefore, we combined EEG and AMRI in healthy human subjects to investigate the role of theta oscillations in coordinating hippocampo-cortical and striatal interactions during contextual retrieval. Participants were familiarized with a set of scene images outside of the scanner and subsequently performed a remember/know recognition task using those images intermixed with new ones while fMRI and EEG data were recorded simultaneously. Frequency-specific power (EEG) and BOLD data (fMRI) were compared between items that were correctly and certainly remembered with contextual information (REC), items that were correctly and certainly identified as old without any recollective experience (FAM), and items that were correctly and certainly identified as new (NEW). We found recollection-specific increases in theta-alpha (4-13 $\mathrm{Hz}$ ) power and a seed region in the hippocampus for which recollection-specific connectivity was estimated using psychophysiological interaction (PPI). Importantly, theta-alpha power correlated with hippocampal connectivity in the PFC and the striatum. These results provide empirical evidence supporting theoretical assumptions about a link between low-frequency oscillations and hippocampal connectivity patterns during recol-
Table 1. RT for old/new responses and mean corrected recollection and familiarity rates

\begin{tabular}{llll}
\hline & REC & FAM & NEW \\
\hline RT (ms) & $1173(167)$ & $1393(183)$ & $1416(233)$ \\
Corrected hit rates (\%) & $39.1(15.8)$ & $20.8(12.6)$ & - \\
\hline
\end{tabular}

Mean values $( \pm S D)$ are given based on $n=20$ subjects. For mean $R T$, there was a main effect of condition $\left(F_{(2,38)}=\right.$ 20.17, $\left.p<0.001, \eta^{2}=0.515, \epsilon=0.716\right)$. Responses were faster for REC compared with NEW $\left(t_{(19)}=5.03, p<\right.$ $0.001)$ and FAM $\left(t_{(19)}=8.56, p<0.001\right)$. There was no difference between FAM and NEW $\left(t_{(19)}=0.47\right.$, $p=0.645$ )

lection and they implicate the PFC and the striatum in cognitive control processes of hippocampus-dependent context retrieval.

\section{Materials and Methods}

Participants. Twenty-seven healthy human subjects participated in the experiment. Due to technical problems, the experiment could not be completed or data storage failed for three subjects. Furthermore, we set a minimal trial criterion within each condition of interest of 15 trials that led to the exclusion of another four subjects before preprocessing, leaving a sample of 20 subjects ( 11 female; age range: $21-31$ years; mean age = 25.1 years; $S D=2.63$ years). During preprocessing, one subject had to be excluded from fMRI analyses because of noisy data that led to an analysis mask lacking large portions of the cortex. The same subject and one additional subject had to be excluded from analysis of frequency-specific power due to a dropping of trial count below the cutoff after artifact rejection. Therefore, the final sample included 19 subjects for fMRI analysis and 18 subjects for power and combined analysis. All subjects were right-handed and had (corrected-to) normal vision. None of the participants reported a history of neurological or psychiatric disorders. The 


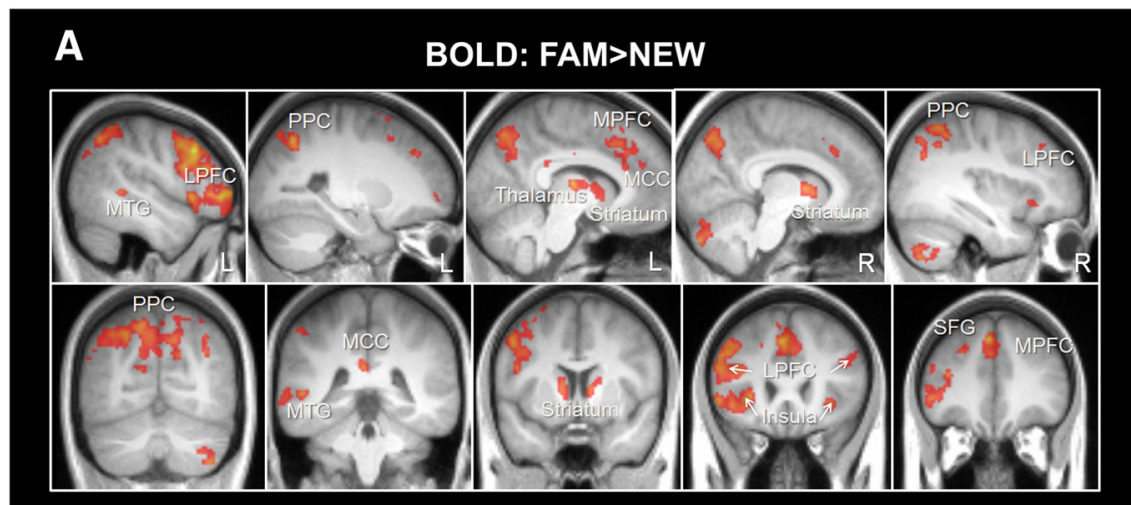

B

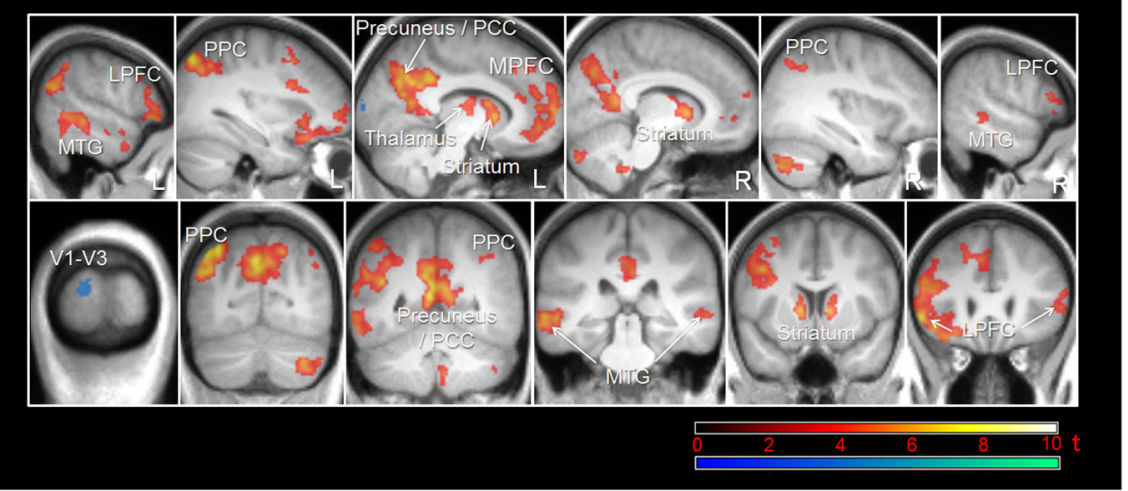

Figure 2. Retrieval success effects in the fMRI data. $A$, Thresholded SPM $\{t\}$ map $\left(p_{(\mathrm{FWE})}<0.05\right)$ for the BOLD contrast FAM $>$ NEW overlaid on the mean T1 image. Slices are placed at $x=-48,-25,-7,10$, and 36 and $y=-65,-40,7,24$, and 36 . $\boldsymbol{B}$, Thresholded SPM $\{t\}$ map $\left(p_{\text {(FWE) }}<0.05\right)$ for the BOLD contrast REC $>$ NEW (red) and REC $<$ NEW (blue) overlaid on the mean T1 image. Slices are placed at $x=-55,-32,-10,10,36$, and 58 and $y=-100,-72,-52,-31,11$, and 26. LPFC, Lateral PFC; MCC, middle cingulate cortex; $\mathrm{PCC}$, posterior cingulate cortex; $\mathrm{SFG}$, superior frontal gyrus; $\mathrm{V1}-\mathrm{V} 3$, visual cortex.

study was approved by the local ethics committee (Medical Council Hamburg) and subjects gave written informed consent.

Experimental design and task. The experiment consisted of a learning phase outside of the MRI scanner, followed by a recognition memory task inside of the scanner. Participants were aware of the recognition task before the learning phase, but were instructed not to use specific memory techniques.

During the learning phase, participants were presented with 75 indoor and 75 outdoor images twice in random order for $1.5 \mathrm{~s}$ with a jittered interstimulus interval (ISI) of $1.5 \pm 0.25 \mathrm{~s}$, during which a fixation cross was presented in the center of the screen. The participants indicated indoor/outdoor status of each image while it was on the screen with a button press of either the index (indoor) or middle (outdoor) finger of the right hand.

Recognition memory for the presented pictures was tested $\sim 45 \mathrm{~min}$ later in two experimental blocks, each consisting of 75 images from the learning phase ("old images") and 75 images that participants had not been presented with previously ("new images"). Memory was tested using a "remember/know" recognition task (Tulving, 1985). Here, all images were randomly intermixed with new images and presented with the query "Old/New/-" written below the image. Participants made this initial "old/new" decision using their right index and middle finger, respectively. After $3.5 \mathrm{~s}$, the image disappeared and, after a "new" decision, subjects were asked to indicate whether they were confident or not ("Sure/Guess/-") that the image had not been presented before, again using the right index and middle finger. Only confident responses were included in the analysis (NEW); guesses were not considered. After an "old" decision, subjects indicated whether they remembered something specific about seeing the image during encoding (REC), simply felt familiarity with the image without recollective experience (FAM), or were guessing that they had seen the image in the learning phase ("Remember/
Know/Guess") using their right index, middle, and ring finger. Importantly, participants were instructed to only respond with "remember" or "know" if they were sure that the image had been presented before. Again, participants had $3.5 \mathrm{~s}$ to indicate their response. If no response had been given in time for either the first or the second judgment, no response was collected for that item and participants were instructed to react faster on subsequent trials. After the second response, there was a jittered ISI of $1.5 \pm 0.25 \mathrm{~s}$ before the next image was presented, during which a white fixation cross was presented in the center of the screen. Participants could make a self-paced pause between blocks and there was another pause of $20 \mathrm{~s}$ after half of the images in each block had been presented.

Before each phase of the experiment, participants completed a training session outside of the MRI scanner. The first training session prepared participants for the learning phase and included 10 indoor and 10 outdoor images. The second training session was conducted after the learning phase of the main experiment was completed and consisted of 10 old and 10 new images. Images used during the training phase were different from those used during testing.

Behavioral data analysis. Corrected hit rates are provided based on the assumption that recollection and familiarity rely on independent processes (Yonelinas and Jacoby, 1995; Yonelinas, 2002). Corrected recollection rates (CRs) were calculated as the probability of making a "remember" judgment to an old item (R), corrected for the probability of making a "remember" judgment to a new item (false alarm rate for "remember" responses $[\mathrm{Fa} \mathrm{R}] ; \mathrm{CR}=\mathrm{R}-$ Fa R). Familiarity rates were corrected for false alarms and the fact that "know" responses were given in the absence of recollection (by dividing by the probability of not remembering context, i.e., $1-\mathrm{CR}$ ). Therefore, corrected familiarity rates (CFs) were calculated as the probability of making a "know" judgment to an old item (K), corrected for the probability of making a "know" judgment to a new item (false alarm rate for "know" responses $[\mathrm{Fa} \mathrm{K}])$ divided by $1-\mathrm{CR}(\mathrm{CF}=$ $[\mathrm{K}-\mathrm{Fa} \mathrm{K}] /[1-\mathrm{CR}])$.

MRI data acquisition and preprocessing. Acquisition of MRI data was performed using a $3 \mathrm{~T}$ Siemens Trio MRI scanner. For functional imaging, 48 slices (aligned to the anteroposterior axis) covering the whole brain were collected per volume using a $\mathrm{T} 2^{\star}$-weighted EPI sequence (continuous slice acquisition, spatial resolution: $2 \times 2 \times 2 \mathrm{~mm}$; slice gap: $1 \mathrm{~mm}$; TR $=4000 \mathrm{~ms}$; TE $=25 \mathrm{~ms}$; flip angle: $90^{\circ}$ along the anteroposterior axis). Images were acquired in two runs. At the beginning of each run, at least five initial volumes were recorded to allow for steady-state magnetization, which were excluded from the analyses.

At the end of the experiment, high-resolution anatomical T1 images of each subject's brain were obtained using MP-RAGE (magnetization prepared gradient echo) acquisition at $1 \mathrm{~mm}^{3}$. For one subject, a T1 image was already available from a study conducted a month earlier and therefore not acquired again.

MRI data were preprocessed and analyzed using SPM12b (http:// www.fil.ion.ucl.ac.uk/spm/software/spm12/) running in MATLAB R2014a (The MathWorks). The origin was manually reset to the anterior commissure to match the template used during normalization. EPI images were then realigned between and within sessions to the first image in the time series using a least-squares approach and a six-parameter rigid body transformation. In the same step, images were unwarped to remove residual movement related variance. Subsequently, the T1-weighted anatomical scan was coregistered to the functional images, again using a rigid 
body transformation. All images were then normalized to MNI space based on normalization parameters derived from a segmentation of T1-weighted images into white matter, gray matter, and CSF using default tissue probability maps. Finally, all images were smoothed using a 6 mm FWHM Gaussian kernel.

MRI statistical analyses. Onset regressors in the first level were included for the three conditions: REC, FAM, and NEW. All images not included in these categories [images incorrectly classified during encoding or retrieval, guesses, and very slow responses during retrieval, reaction time $(\mathrm{RT})>3 \mathrm{~s}$ ] were subsumed in one regressor of no interest. Stick functions were convolved with the canonical HRF and a high-pass filter (cutoff $1 / 128 \mathrm{~Hz}$ ) was applied. Runs were concatenated for first-level analysis due to an unbalanced/low number of trials per run within conditions of interest for some subjects. High-pass filter, block constants, and autoregressive models were adjusted to be block specific. Contrasts for each condition of interest versus the implicit baseline were entered into a within-subjects ANOVA with memory (REC, FAM, or NEW) as the only factor on the second level. The uncorrected $p$-threshold at the voxel level used for clustering for this and all following analyses was $p<0.001$. FWE correction was used to account for the multiple-comparisons problem. Clusters were considered significant at $p_{(\mathrm{FWE})}<0.05$ at the cluster level or peak voxel.

For the PPI analysis, the first eigenvariate (adjusted for the $F$ contrast of interest) was extracted from a subject-invariant volume of interest based on the effect in the right hippocampus for the contrast REC > FAM (the significant cluster was masked to include only the hippocampus). An interaction of the eigenvariate and the contrast REC $>$ FAM was calculated and used as a regressor in the first-level analysis. Additional regressors of no interest were included to account for non-task-related effects (main effect of the extracted signal from the hippocampus) and correlations that are driven only by task input (main effect of the experimental contrast REC $>$ FAM) to ensure that variance explained by the interaction is only that over and above what is explained by the main effects of physiological and psychological variable. In addition, the onset regressors described above were included to model variance due to the task and render the analysis maximally sensitive (O'Reilly et al., 2012). Again, runs were concatenated (see above). A one-sample $t$ test was conducted at the second level to test for the PPI effect.

EEG data acquisition and preprocessing. EEG data were acquired simultaneously with 64 electrodes (sintered silver and silver-chloride) placed on the scalp using a custom-built electrode system (Braincap MR; Brain Products). Fz was used as a reference electrode and $\mathrm{POz}$ as a ground electrode during recording. Electrode skin impedances were usually $<5 \mathrm{k} \Omega$. Two additional electrodes were placed on the participant's back and beneath the left eye, which were used to record electrocardiogram and eye movements, respectively. Data were recorded with a sampling rate of $5000 \mathrm{~Hz}$ and a resolution of $0.5 \mu \mathrm{V}$ and online highpass (cutoff $0.016 \mathrm{~Hz}$ ) and low-pass (cutoff 250 $\mathrm{Hz}$ ) filtered using the BrainVision Recorder 2.0 (Brain Products).

MR gradient correction was performed using BrainVision Analyzer 2.0 (Brain Products) by subtracting a sliding average over 21 epochs time locked to the scanner pulses (excluding the dummy scans) and baseline corrected over the entire epoch. Data were low-pass filtered using a finite impulse response (FIR) filter with a cutoff of $70 \mathrm{~Hz}$ and then exported to EEGLAB (Delorme and Makeig, 2004). The FMRIB plug-in for EEGLAB, provided by the University of Oxford Centre for Functional MRI of the Brain (FMRIB) (Iannetti et al., 2005; Niazy et al., 2005), was used to attenuate the balistocardiographic (BCG) artifact, which is caused by an induction of potentials through blood moving within the magnetic field during the cardiac cycle, as well as associated head and scalp movement (Allen et al., 1998; Debener et al., 2008). For that, QRS peaks in the ECG channel were
Table 2. Peak-level and cluster statistics for the contrast FAM > NEW

\begin{tabular}{|c|c|c|c|c|c|c|c|}
\hline \multicolumn{3}{|l|}{$\overline{\text { Cluster }}$} & \multicolumn{5}{|l|}{ Peak } \\
\hline Label & $k$ & $p_{(\mathrm{FWE})}$ & $t$ & $p_{(\mathrm{FWE})}$ & $x$ & $y$ & $z$ \\
\hline LLPFC/insula & 4079 & $<0.001$ & 8.80 & $<0.001$ & -46 & 20 & 36 \\
\hline L striatum/thalamus & 294 & $<0.001$ & 6.86 & 0.005 & -12 & 12 & 4 \\
\hline B MPFC/cingulate & 1121 & $<0.001$ & 6.56 & 0.013 & -4 & 34 & 44 \\
\hline B PPC & 2873 & $<0.001$ & 6.48 & 0.016 & -26 & -64 & 46 \\
\hline$R$ cerebellum & 218 & $<0.001$ & 6.22 & 0.036 & 12 & -84 & -38 \\
\hline R striatum & 194 & $<0.001$ & 5.84 & 0.101 & 10 & 4 & 6 \\
\hline BMCC & 335 & $<0.001$ & 5.84 & 0.101 & -2 & -38 & 22 \\
\hline LMTG & 220 & $<0.001$ & 5.76 & 0.121 & -50 & -42 & 2 \\
\hline RPPC & 293 & $<0.001$ & 5.47 & 0.240 & 36 & -54 & 56 \\
\hline R LPFC & 225 & $<0.001$ & 5.40 & 0.276 & 44 & 28 & 30 \\
\hline R cerebellum & 245 & $<0.001$ & 5.40 & 0.276 & 34 & -70 & -50 \\
\hline Rinsula & 105 & 0.010 & 5.35 & 0.307 & 34 & 22 & -6 \\
\hline LSFG & 120 & 0.005 & 4.66 & 0.870 & -24 & 38 & 34 \\
\hline LIPL & 85 & 0.029 & 4.58 & 0.916 & 36 & -66 & 34 \\
\hline
\end{tabular}

IPL, Inferior parietal lobule; MCC, midcingulate cortex; SFG, superior frontal gyrus.

Table 3. Peak-level and cluster statistics for the contrast REC $>$ NEW and REC $<$ NEW

\begin{tabular}{|c|c|c|c|c|c|c|c|}
\hline \multicolumn{3}{|l|}{ Cluster } & \multicolumn{5}{|l|}{ Peak } \\
\hline Label & $k$ & $p_{(\mathrm{FWE})}$ & $t$ & $p_{(\mathrm{FWE})}$ & $x$ & $y$ & $z$ \\
\hline \multicolumn{8}{|l|}{$\mathrm{REC}>\mathrm{NEW}$} \\
\hline B precuneus/PCC & 4284 & $<0.001$ & 8.48 & $<0.001$ & -6 & -50 & 12 \\
\hline LPPC & 2352 & $<0.001$ & 7.91 & $<0.001$ & -32 & -72 & 48 \\
\hline L LPFC and B MPFC/ACC & 7710 & $<0.001$ & 7.31 & 0.001 & -52 & 28 & -4 \\
\hline L striatum/thalamus & 395 & $<0.001$ & 6.77 & 0.007 & -12 & 12 & 4 \\
\hline $\mathrm{R}$ cerebellum & 782 & $<0.001$ & 6.59 & 0.011 & 32 & -74 & -38 \\
\hline L MTG & 1172 & $<0.001$ & 6.52 & 0.014 & -64 & -32 & -4 \\
\hline R striatum & 343 & $<0.001$ & 6.45 & 0.017 & 10 & 10 & 2 \\
\hline Brainstem & 150 & 0.001 & 5.97 & 0.072 & -4 & -26 & -26 \\
\hline$R$ cerebellum & 104 & 0.011 & 5.85 & 0.097 & 6 & -48 & -44 \\
\hline R LPFC & 161 & 0.001 & 5.35 & 0.312 & 56 & 22 & 16 \\
\hline RPPC & 225 & $<0.001$ & 4.82 & 0.751 & 36 & -54 & 42 \\
\hline R MTG & 108 & 0.009 & 4.58 & 0.916 & 62 & -30 & -2 \\
\hline \multicolumn{8}{|l|}{ REC $<$ NEW } \\
\hline V1-V3 & 89 & 0.023 & 4.77 & 0.791 & -14 & -100 & 10 \\
\hline
\end{tabular}

ACC, Anterior cingulate cortex; V1-V3, visual cortex.

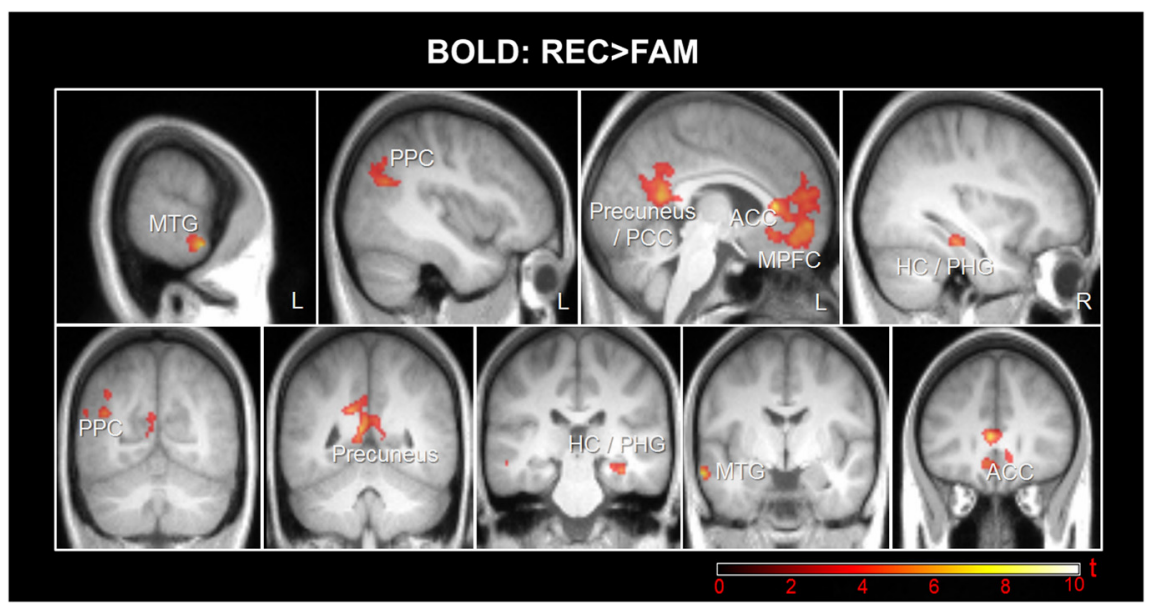

Figure 3. Recollection-specific increase in BOLD. Thresholded SPM $\{t\}$ map $\left(p_{(\text {FWE })}<0.05\right)$ for the contrast REC $>$ FAM overlaid on the mean $\mathrm{T} 1$ image. Slices are placed at $x=-64,-40,-5$, and 34 and $y=-62,-50,-28,-6$, and 30. ACC, Anterior cingulate cortex; HC, hippocampus; PCC, posterior cingulate cortex; PHG, parahippocampal gyrus. detected with the algorithm implemented in the FMRIB plugin and controlled visually. When automatic detection did not lead to satisfactory detection accuracy, QRS events were semiautomatically set in BrainVision Analyzer. After QRS detection was completed, data were down- 
Table 4. Peak-level and cluster statistics for the contrast REC > FAM

\begin{tabular}{|c|c|c|c|c|c|c|c|}
\hline \multicolumn{3}{|l|}{ Cluster } & \multicolumn{5}{|l|}{ Peak } \\
\hline Label & $k$ & $p_{(\mathrm{FWE})}$ & $t$ & $p_{\text {(FWE) }}$ & $x$ & $y$ & $Z$ \\
\hline L MTG & 286 & $<0.001$ & 7.14 & 0.002 & -64 & -6 & -18 \\
\hline B MPFC/ACC & 1971 & $<0.001$ & 7.13 & 0.002 & -4 & 30 & 8 \\
\hline B precuneus/PCC & 801 & $<0.001$ & 6.34 & 0.024 & -6 & -50 & 12 \\
\hline RHC/PHG & 90 & 0.022 & 5.26 & 0.371 & 34 & -28 & -16 \\
\hline LPPC & 310 & $<0.001$ & 5.20 & 0.415 & -40 & -62 & 24 \\
\hline
\end{tabular}

ACC, Anterior cingulate cortex; $\mathrm{HC}$, hippocampus; PHG, parahippocampal gyrus.

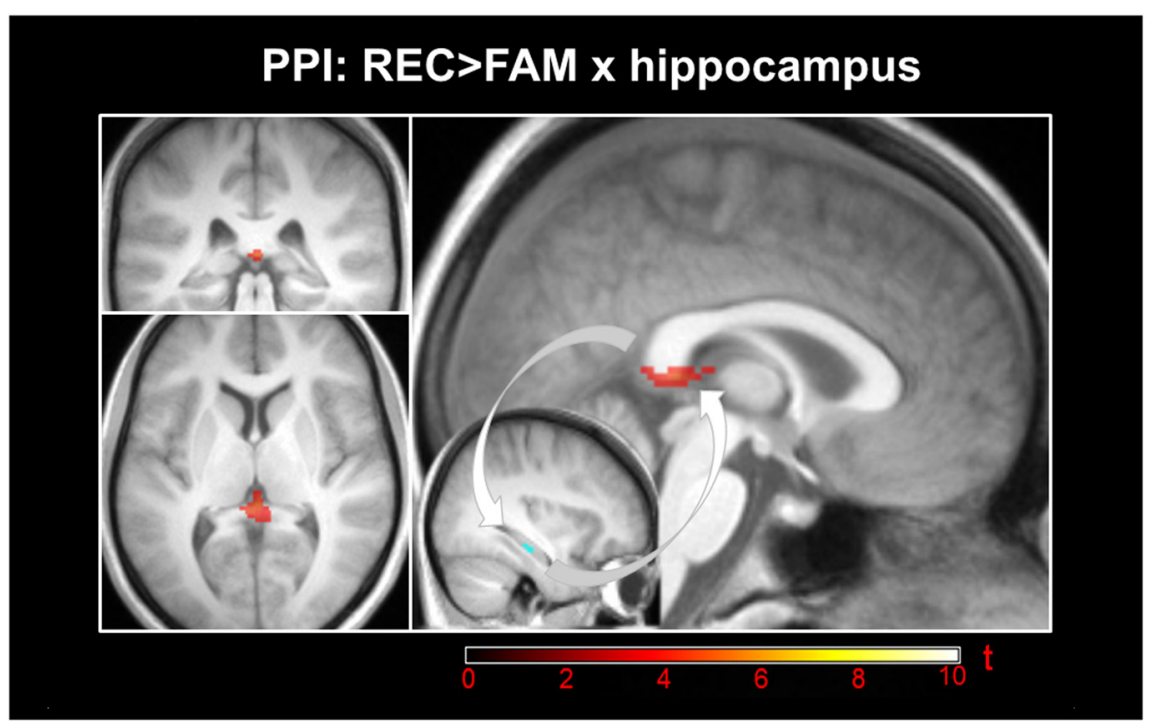

Figure 4. Hippocampal connectivity during recollection. Thresholded SPM $\{t\} \operatorname{map}\left(p_{(\mathrm{FWE})}<0.05\right)$ for the PPI of right hippocampus $\times$ REC $>$ FAM together with the hippocampal seed region overlaid on the mean T1 image. A recollection-specific increase in hippocampal contribution is located between the posterior end of the cingulate cortex, the splenium, and the fornix, possibly including the retrosplenial cortex. The cluster is adjacent to (and slightly overlapping with) the PCC/precuneus cluster found in the contrast REC $>$ NEW (Fig. 2, Table 3). This effect is consistent with the suggestion that the retrosplenial cortex relays information from the hippocampus to the cortex, driving reinstatement of encoding related representations (Johnson and Rugg, 2007).

Table 5. Peak-level and cluster statistics for the PPI (right hippocampus $\times$ REC $>$ FAM)

\begin{tabular}{|c|c|c|c|c|c|c|c|}
\hline \multicolumn{3}{|c|}{ Cluster } & \multicolumn{5}{|l|}{ Peak } \\
\hline Label & $k$ & $p_{(\mathrm{FWE})}$ & $t$ & $p_{(\mathrm{FWE})}$ & $x$ & $y$ & Z \\
\hline$P C C$ & 72 & 0.025 & 4.90 & 0.998 & 2 & -34 & 6 \\
\hline
\end{tabular}

$\mathrm{PCC}$, Posterior cingulate cortex.

sampled to $500 \mathrm{~Hz}$. For the actual correction, EEG data from each channel were separately aligned to the BCG artifact (based on the detected QRS peaks) in a matrix to perform principal component analysis. The first four principal components were used as an optimal basis set describing the artifact, which was then fitted to and subtracted from each QRS event segment (Niazy et al., 2005). Subsequently, data were sequentially low-pass (cutoff $40 \mathrm{~Hz}$ ) and high-pass (cutoff $0.5 \mathrm{~Hz}$ ) filtered using a FIR filter and then exported to Fieldtrip (Oostenveld et al., 2011) for all further analysis steps. Here, data were epoched from $1 \mathrm{~s}$ before to $2 \mathrm{~s}$ after stimulus onset. Trials were mean corrected and screened visually for major atypical artifacts (originating most likely from movement) and bad channels (no more than a single channel had to be excluded for any subject) before performing independent component analysis individually for each run. On average, four components were identified as being artifactual (representing mainly blinks and eye movements) by visual inspection and rejected per subject and run. All other components were back projected to electrode space and previously excluded channels were interpolated using spherical spline interpolation (Perrin et al., 1989). Epochs were re-referenced to average reference and a threshold of \pm 150 $\mu \mathrm{V}$ was applied to reject residual artifacts within the analysis time window between -1 and $2 \mathrm{~s}$ with respect to stimulus onset.

EEG statistical analyses. Time-frequency decomposition and statistical analysis were performed using Fieldtrip (Oostenveld et al., 2011) running in MATLAB 2014a (The MathWorks). Time-frequency decomposition was done using convolution with complex morlet wavelets ( 4 cycles) in the frequency range within 4 to $30 \mathrm{~Hz}$ with a resolution of $0.33 \mathrm{~Hz}$ in the frequency and $10 \mathrm{~ms}$ in the time domain. Power was averaged across trials within the conditions of interest and averaged data were baseline corrected using a condition-specific relative baseline $(-0.5$ to $-0.1 \mathrm{~s}$ prestimulus). Power was analyzed with a nonparametric cluster-based permutation test (Maris and Oostenveld, 2007) in a time window from 0 to $1.5 \mathrm{~s}$. For all contrasts, a $t$ test was conducted for each individual sample in 3D space (channel, frequency, time). Clusters were formed by adjacent significant samples $(p<$ 0.05). The neighborhood definition for channels was based on triangulation and only effects significant at three or more neighboring channels were considered in the clustering algorithm. To control for multiple comparisons, a Monte Carlo estimate of the permutation $p$-value was calculated by randomly permuting condition labels $(n=5000)$ and comparing the clusters statistic (sum of $t$-values) of each cluster found in real data with that found in surrogate data. The proportion of cluster statistics in surrogate data exceeding that in real data gives the $p$-value. Clusters were considered significant at $p<0.05$.

Combined statistical analysis: power and $P P I$. For the correlation of the recollectionspecific increase in theta-alpha power with the recollection-specific increase in hippocampal connectivity, the difference in theta-alpha power (REC - FAM) was extracted for each subject using the group-level mask of the identified cluster (REC $>$ FAM) and averaged across samples. This variable was then used as a covariate in the second-level $t$ test on the PPI (see above). No outlier values (mean $\pm 3{ }^{\star} \mathrm{SD}$ ) were identified in the covariate. Two separate small volume corrections (SVCs) were applied: one in the PFC (including superior, middle, medial, and inferior frontal gyri) and one in the caudate. Masks were generated using the TD atlas in the WFU Pickatlas toolbox (Lancaster et al., 1997, 2000; Maldjian et al., 2003).

Trial count for all analyses. The mean number of trials within conditions of interest for fMRI analyses ( $n=19$ subjects) was 58.5 for REC, 43.6 for FAM, and 79.0 for NEW. Artifact rejection in the EEG data reduced the number of trials for EEG and combined analysis compared with $\mathrm{fMRI}$ analysis. For power analyses and combined analysis of power and BOLD ( $n=18$ subjects), the mean number of trials was 51.4 for REC, 39.4 for FAM, and 71.2 for NEW. As mentioned above, the minimum number of trials per subject and condition was 15 for all analyses.

\section{Results}

Regarding the EEG data, frequency-specific power time locked to stimulus onset was analyzed using cluster-based permutation in 3D space (time, frequency, channel). We identified a cluster broadly distributed across channels in the alpha and theta frequency band $(4-13 \mathrm{~Hz})$ spanning from 490 to $1500 \mathrm{~ms}$ in the lowest frequency (Fig. 1A) differentiating significantly between REC and FAM $(p=0.006)$. As expected, this effect was driven by an increase in theta-alpha power relative to baseline, which was stronger for REC than FAM (Fig. 1B). There was no statistically significant cluster expressing the opposite effect (REC $<$ FAM; $p=0.09)$, and no cluster reached significance for the contrasts REC $>\operatorname{NEW}(p=0.12)$, REC $<$ NEW $(p=0.14)$, FAM $>$ NEW 
$(p=1)$, and FAM $<\operatorname{NEW}(p=0.10)$. For behavioral data, see Table 1 .

Regarding the fMRI data, we found retrieval success effects (FAM $>$ NEW and/or REC $>$ NEW, $p_{(\mathrm{FWE})}<0.05$; Fig. 2, Table 2, Table 3) in bilateral medial PFC (MPFC) and precuneus (extending into anterior and posterior cingulate, respectively), lateral PFC, posterior parietal cortex (PPC), middle temporal gyrus (MTG), striatum (caudate), and left thalamus. An effect for the contrast REC $<$ NEW was found in the visual cortex (Fig. 2, Table 3). Importantly, specific for recollection of contextual information (REC > FAM), we found significant activation in MPFC and precuneus (extending into anterior and posterior cingulate, respectively), left PPC, left MTG, and right MTL (hippocampus and adjacent posterior parahippocampal gyrus) $\left(p_{\text {(FWE) }}<0.05\right.$; Fig. 3 , Table 4). These findings are consistent with previous studies (Spaniol et al., 2009).

To further assess the functional properties of the underlying network, we first used the part of the right hippocampus discriminating between REC and FAM as a seed region for the PPI analysis. Here, greater contribution of the hippocampus for REC than FAM $\left(p_{\text {(FWE) }}<0.05\right)$ was identified in a cluster located between the posterior end of the cingulate, the splenium, and the fornix, possibly including the retrosplenial cortex (Fig. 4, Table 5). In a second analysis, we were specifically interested in the link between hippocam-

pal connectivity and theta-alpha power. Therefore, the difference in theta-alpha power between REC and FAM was extracted from the identified cluster mentioned above (EEG analysis), averaged across samples for each subject, and correlated with the recollection-specific increase in hippocampal connectivity across the whole brain. We found one cluster in the left caudate and two clusters in the left PFC, one located in BA 47, in the depth of the anterior part of the superior frontal sulcus, and one located in the (medial) superior frontal gyrus at the intersection of BA 32 and BA 10 showing a significant correlation (Fig. 5, Table 6). All three clusters survived correction for multiple comparisons $\left(p_{(\mathrm{FWE})}<\right.$ $0.05)$ using SVC within a priori defined regions of interest: the PFC and the caudate, which is the part of the striatum that showed reliable retrieval success effects in the current study (Fig. 2, Table 2, Table 3) and in previous studies (Spaniol et al., 2009).

An additional within-subjects analysis did not reveal any significant effects. Here, a parametric modulation with theta-alpha power per trial was used as the psychological variable in a PPI analysis using the gPPI Toolbox (McLaren et al., 2012) and contrasted for REC and FAM.

\section{Discussion}

By combining EEG and fMRI during a remember/know recognition memory task, we could replicate a link between theta(-alpha) power and recollection of contextual information (Fig. 1A; Guderian and Düzel, 2005) and a recollection-specific network of brain areas
Table 6. Peak-level and cluster statistics for the correlation of recollection-specific connectivity with the hippocampus and theta-alpha power

\begin{tabular}{|c|c|c|c|c|c|c|}
\hline \multicolumn{2}{|l|}{ Cluster } & \multicolumn{5}{|l|}{ Peak } \\
\hline Label & $k$ & $t$ & $p_{(\mathrm{SVC}, \mathrm{FWE})}$ & $x$ & $y$ & $z$ \\
\hline LLPFC & 18 & 7.78 & 0.014 & -28 & 42 & -2 \\
\hline L MPFC & 17 & 7.20 & 0.038 & -14 & 48 & 22 \\
\hline L caudate & 24 & 5.21 & 0.043 & -10 & 14 & 8 \\
\hline
\end{tabular}

Note that all three peak voxels survived SVC using PFC and caudate as a priori defined regions of interest $\left(p_{\text {(FWE) }}<0.05\right)$.

including the bilateral MPFC, precuneus, left MTG, PPC, and right hippocampus (Fig. 3; Spaniol et al., 2009). Importantly, we can demonstrate that recollection-specific theta-alpha power correlates with recollection-specific increases in hippocampal contribution to activity in the PFC and caudate in a between-subjects analysis (Fig. 5). This means that subjects exhibiting a more pronounced effect in theta-alpha power differentiating between recollection and familiarity also show a more pronounced difference in hippocampal connectivity with PFC and caudate between recollection and familiarity.

A link between theta-alpha power and hippocampal connectivity with the PFC and striatum confirms our main hypothesis and supports the notion of theta oscillations providing a mechanism for long-range coordination of segregated brain regions (Sauseng et al., 2010). Alternatively (but not mutually exclu- 
sively), theta oscillations have been suggested to define an optimal window for encoding or retrieval by modulating excitability and the direction of information flow in hippocampal networks and to organize multiple features into coherent representations (involving temporal and spatial information) by coupling with higher frequencies and phase coding (Hasselmo et al., 2002; Sauseng et al., 2010; Fell and Axmacher, 2011). Interestingly, recollection-specific EEG activity was not restricted to the theta band. Instead, it ranged from 4 to $13 \mathrm{~Hz}$ and therefore included the alpha band. This observation is consistent with previous work suggesting there might not be a clear functional distinction between the alpha- and theta-band in memory functions. For instance, theta- and alpha-power similarly increase during working memory maintenance in scalp-recorded EEG (Eckart et al., 2014), whereas the peak frequency is subject to large interindividual differences and varies between 2 and $18 \mathrm{~Hz}$ (Cohen, 2011). Moreover, during free recall of verbal episodic memories, intracranial recordings revealed increases of theta-power similarly extending up to 13-15 Hz (Burke et al., 2014).

Consistent with our current results, differences in theta power between recollection and familiarity have previously been source localized to the MTL and the PFC, but, importantly, not deeper sources (Guderian and Düzel, 2005). Striatal contributions to mnemonic functions - especially retrieval - have only recently received great attention (Scimeca and Badre, 2012; Clos et al., 2015). In general terms, the striatum may act as a highly selective filter (i.e., gate) for representations to be encoded in working memory (Frank et al., 2001; O'Reilly and Frank, 2006) or stored in long-term memory (Lisman and Grace, 2005). Physiologically, and consistent with the current findings, it receives input from the hippocampus (Laroche et al., 2000), providing information about item novelty and stored associations (Lisman and Grace, 2005). During retrieval, there are different possible functions of hippocampostriatal interactions. Most prominently, a loop including the hippocampus and striatum might strengthen a re-encoding of items with high utility (based on the fact that utility influences the probability of retrieval, retrieval itself might be used as a utility cue; Lisman and Grace, 2005; Scimeca and Badre, 2012). Alternatively, hippocampostriatal interactions might drive memory-based decision making (Johnson et al., 2007; Wimmer and Shohamy, 2012) or reinforcement of successful retrieval strategies (Scimeca and Badre, 2012). Finally, the striatum might gate working memory content during retrieval (Scimeca and Badre, 2012), meaning that it might select retrieval cues that are maintained by the PFC to bias matching processes in the hippocampus or ultimately gate contextual information stored in hippocampal patterns into working memory providing the consciously accessible sensation of remembering.

Within the PFC, a correlation of theta-alpha power with hippocampal connectivity was found with high spatial precision in the depth of the superior frontal sulcus at the intersection of lateral and anterior PFC (BA 47) and in the (medial) superior frontal gyrus at the intersection of medial and anterior PFC (BA 32 and BA 10). As such, these results go beyond previous MEG studies (Guderian and Düzel, 2005) and are in good agreement with work implicating the PFC in episodic retrieval (Gilbert et al., 2006; Han et al., 2010), recollection (Rugg et al., 1999; Dobbins et al., 2002; Simons et al., 2005; Spaniol et al., 2009), retrieval mode (Lepage et al., 2000), mental imagery (Mechelli et al., 2004), and visual working memory (Ranganath, 2006). However, the precise functions of prefrontal areas in mnemonic processes remain debated with suggestions ranging from specification of retrieval cues and strategies (Dobbins et al., 2002), top-down control on sensory areas coding specific item features (Ranganath, 2006), integration of new information with semantic knowledge (Preston and Eichenbaum, 2013), planning of retrieval-based actions (Han et al., 2010) and prospective remembering (Reynolds et al., 2009), to evaluation of retrieved and processing of rewardrelated or self-relevant information (Simons and Spiers, 2003).

There are at least two possible explanations for a correlation of hippocampal connectivity with theta power in the PFC and striatum. First, connectivity and information transfer between these areas might be facilitated by or reflected in coordinated theta oscillations within the very same areas. Alternatively, connectivity between these areas might itself be independent of theta but enhance synchronization in the theta range at (distinct) neocortical sites involved in subcomponents of recollection (e.g., reinstatement of sensory features). Theta rhythms are known to be generated in the hippocampal formation, where their crucial role in memory and spatial and temporal organization is well investigated (Buzsáki, 2002). Hippocampal theta is known to interact with theta phase and spike timing in the striatum (DeCoteau et al., 2007; van der Meer and Redish, 2011) and (medial) PFC (Hyman et al., 2005; Jones and Wilson, 2005; Siapas et al., 2005; Benchenane et al., 2011), which is consistent with the former account. However, there are also widespread neocortical areas in temporal, parietal, occipital (Raghavachari et al., 2006), and frontal (Cantero et al., 2003) cortex generating local theta fluctuations. Consistent with the latter account, intracranial recordings during free recall showed enhanced theta-power in anterior temporal cortex, but not the MTL, where high-frequency activity between 64 and $96 \mathrm{~Hz}$ was prevalent (Burke et al., 2014). Moreover, scalp EEG is particularly sensitive to activity from neocortical areas rather than deep sources such as the hippocampus and striatum and the scalp distribution that we observed was most strongly pronounced at occipitoparietal electrodes, making the latter account seem equally plausible. Finally, it should be noted that both accounts are not mutually exclusive and the two mechanisms could concurrently contribute to the observed effects.

\section{Conclusion}

For the first time in humans, we can show that scalp-recorded theta-alpha power is linked to hippocampal connectivity with the striatum and PFC. These results provide compelling evidence in favor of theoretical models of recollection implicating the hippocampus in coordinating neocortical activity via low-frequency oscillations. Moreover, they implicate the PFC and the striatum in cognitive control processes of hippocampus-dependent contextual retrieval and link these control processes to theta and alpha oscillations. Finally, they bridge the gap between results from neuroimaging and neurophysiology and, from a more general point of view, further indicate that combining BOLDderived connectivity measures with oscillatory synchrony may be a powerful mean to investigate neural mechanisms with high spatial and temporal precision.

\section{References}

Allen PJ, Polizzi G, Krakow K, Fish DR, Lemieux L (1998) Identification of EEG events in the MR scanner: the problem of pulse artifact and a method for its subtraction. Neuroimage 8:229-239. CrossRef Medline

Battaglia FP, Benchenane K, Sirota A, Pennartz CM, Wiener SI (2011) The hippocampus: hub of brain network communication for memory. Trends Cogn Sci 15:310-318. Medline

Benchenane K, Tiesinga PH, Battaglia FP (2011) Oscillations in the prefron- 
tal cortex: a gateway to memory and attention. Curr Opin Neurobiol 21:475-485. CrossRef Medline

Burke JF, Sharan AD, Sperling MR, Ramayya AG, Evans JJ, Healey MK, Beck EN, Davis KA, Lucas TH 2nd, Kahana MJ (2014) Theta and highfrequency activity mark spontaneous recall of episodic memories. J Neurosci 34:11355-11365. CrossRef Medline

Buzsáki G (2002) Theta oscillations in the hippocampus. Neuron 33: 325-340. CrossRef Medline

Cantero JL, Atienza M, Stickgold R, Kahana MJ, Madsen JR, Kocsis B (2003) Sleep-dependent theta oscillations in the human hippocampus and neocortex. J Neurosci 23:10897-10903. Medline

Clos M, Schwarze U, Gluth S, Bunzeck N, Sommer T (2015) Goal- and retrieval-dependent activity in the striatum during memory recognition. Neuropsychologia 72:1-11. CrossRef Medline

Cohen MX (2011) Hippocampal-prefrontal connectivity predicts midfrontal oscillations and long-term memory performance. Curr Biol 21:1900-1905. CrossRef Medline

Colgin LL (2013) Mechanisms and functions of theta rhythms. Annu Rev Neurosci 36:295-312. CrossRef Medline

Debener S, Mullinger KJ, Niazy RK, Bowtell RW (2008) Properties of the ballistocardiogram artefact as revealed by EEG recordings at 1.5, 3 and $7 \mathrm{~T}$ static magnetic field strength. Int J Psychophysiol 67:189-199. CrossRef Medline

DeCoteau WE, Thorn C, Gibson DJ, Courtemanche R, Mitra P, Kubota Y, Graybiel AM (2007) Learning-related coordination of striatal and hippocampal theta rhythms during acquisition of a procedural maze task. Proc Natl Acad Sci U S A 104:5644-5649. CrossRef Medline

Delorme A, Makeig S (2004) EEGLAB: An open source toolbox for analysis of single-trial EEG dynamics including independent component analysis. J Neurosci Methods 134:9-21. CrossRef Medline

Dobbins IG, Foley H, Schacter DL, Wagner AD (2002) Executive control during episodic retrieval: multiple prefrontal processes subserve source memory. Neuron 35:989-996. CrossRef Medline

Eckart C, Fuentemilla L, Bauch EM, Bunzeck N (2014) Dopaminergic stimulation facilitates working memory and differentially affects prefrontal low theta oscillations. Neuroimage 94:185-192. CrossRef Medline

Fell J, Axmacher N (2011) The role of phase synchronization in memory processes. Nat Rev Neurosci 12:105-118. CrossRef Medline

Frank MJ, Loughry B, O'Reilly RC (2001) Interactions between frontal cortex and basal ganglia in working memory: a computational model. Cogn Affect Behav Neurosci 1:137-160. CrossRef Medline

Fuentemilla L, Barnes GR, Düzel E, Levine B (2014) Theta oscillations orchestrate medial temporal lobe and neocortex in remembering autobiographical memories. Neuroimage 85:730-737. CrossRef Medline

Gilbert SJ, Spengler S, Simons JS, Steele JD, Lawrie SM, Frith CD, Burgess PW (2006) Functional specialization within rostral prefrontal cortex (area 10): a meta-analysis. J Cogn Neurosci 18:932-948. CrossRef Medline

Guderian S, Düzel E (2005) Induced theta oscillations mediate large-scale synchrony with mediotemporal areas during recollection in humans. Hippocampus 15:901-912. CrossRef Medline

Han S, Huettel SA, Raposo A, Adcock RA, Dobbins IG (2010) Functional significance of striatal responses during episodic decisions: recovery or goal attainment? J Neurosci 30:4767-4775. CrossRef Medline

Hasselmo ME, Bodelón C, Wyble BP (2002) A proposed function for hippocampal theta rhythm: separate phases of encoding and retrieval enhance reversal of prior learning. Neural Comput 14:793-817. CrossRef Medline

Hyman JM, Zilli EA, Paley AM, Hasselmo ME (2005) Medial prefrontal cortex cells show dynamic modulation with the hippocampal theta rhythm dependent on behavior. Hippocampus 15:739-749. CrossRef Medline

Iannetti GD, Niazy RK, Wise RG, Jezzard P, Brooks JC, Zambreanu L, Vennart W, Matthews PM, Tracey I (2005) Simultaneous recording of laser-evoked brain potentials and continuous, high-field functional magnetic resonance imaging in humans. Neuroimage 28:708-719. CrossRef Medline

Johnson A, van der Meer MA, Redish AD (2007) Integrating hippocampus and striatum in decision-making. Curr Opin Neurobiol 17:692-697. CrossRef Medline

Johnson JD, Rugg MD (2007) Recollection and the reinstatement of encoding-related cortical activity. Cereb Cortex 17:2507-2515. CrossRef Medline

Jones MW, Wilson MA (2005) Theta rhythms coordinate hippocampal- prefrontal interactions in a spatial memory task. PLoS Biol 3:e402. Medline

Klimesch W, Freunberger R, Sauseng P (2010) Oscillatory mechanisms of process binding in memory. Neurosci Biobehav Rev 34:1002-1014. CrossRef Medline

Lancaster JL, SummerIn JL, Freitas CS, Fox PT (1997) The Talairach Daemon, a database server for Talairach atlas labels. Neuroimage 5:633.

Lancaster JL, Woldorff MG, Parsons LM, Liotti M, Freitas CS, Rainey L, Kochunov PV, Nickerson D, Mikiten SA, Fox PT (2000) Automated Talairach atlas labels for functional brain mapping. Hum Brain Mapp 10:120-131. CrossRef Medline

Laroche S, Davis S, Jay TM (2000) Plasticity at hippocampal to prefrontal cortex synapses: dual roles in working memory and consolidation. Hippocampus 10:438-446. Medline

Lepage M, Ghaffar O, Nyberg L, Tulving E (2000) Prefrontal cortex and episodic memory retrieval mode. Proc Natl Acad Sci U S A 97:506-511. CrossRef Medline

Lisman JE, Grace AA (2005) The hippocampal-VTA loop: controlling the entry of information into long-term memory. Neuron 46:703-713. CrossRef Medline

Maldjian JA, Laurienti PJ, Kraft RA, Burdette JH (2003) An automated method for neuroanatomic and cytoarchitectonic atlas-based interrogation of fMRI data sets. Neuroimage 19:1233-1239. CrossRef Medline

Maris E, Oostenveld R (2007) Nonparametric statistical testing of EEG- and MEG-data. J Neurosci Methods 164:177-190. CrossRef Medline

Mayes A, Montaldi D, Migo E (2007) Associative memory and the medial temporal lobes. Trends Cogn Sci 11:126-135. CrossRef Medline

McLaren DG, Ries ML, Xu G, Johnson SC (2012) Neuroimage A generalized form of context-dependent psychophysiological interactions (gPPI): a comparison to standard approaches. Neuroimage 61:1277-1286. CrossRef Medline

Mechelli A, Price CJ, Friston KJ, Ishai A (2004) Where bottom-up meets top-down: neuronal interactions during perception and imagery. Cereb Cortex 14:1256-1265. CrossRef Medline

Niazy RK, Beckmann CF, Iannetti GD, Brady JM, Smith SM (2005) Removal of FMRI environment artifacts from EEG data using optimal basis sets. Neuroimage 28:720-737. CrossRef Medline

Norman KA, O’Reilly RC (2003) Modeling hippocampal and neocortical contributions to recognition memory: a complementary-learningsystems approach. Psychol Rev 110:611-646. CrossRef Medline

Oostenveld R, Fries P, Maris E, Schoffelen JM (2011) FieldTrip: Open source software for advanced analysis of MEG, EEG, and invasive electrophysiological data. Comput Intell Neurosci 2011:156869. Medline

O’Reilly JX, Woolrich MW, Behrens TE, Smith SM, Johansen-Berg H (2012) Tools of the trade: psychophysiological interactions and functional connectivity. Soc Cogn Affect Neurosci 7:604-609. CrossRef Medline

O’Reilly RC, Frank MJ (2006) Making working memory work: a computational model of learning in the prefrontal cortex and basal ganglia. Neural Comput 18:283-328. Medline

Perrin F, Pernier J, Bertrand O, Echallier JF (1989) Spherical splines for scalp potential and current density mapping. Electroencephalogr Clin Neurophysiol 72:184-187. CrossRef Medline

Preston AR, Eichenbaum H (2013) Interplay of hippocampus and prefrontal cortex in memory. Curr Biol 23:R764-R773. CrossRef Medline

Raghavachari S, Lisman JE, Tully M, Madsen JR, Bromfield EB, Kahana MJ (2006) Theta oscillations in human cortex during a working-memory task: evidence for local generators. J Neurophysiol 95:1630-1638. CrossRef Medline

Ranganath C (2006) Working memory for visual objects: complementary roles of inferior temporal, medial temporal, and prefrontal cortex. Neuroscience 139:277-289. CrossRef Medline

Reynolds JR, West R, Braver T (2009) Distinct neural circuits support transient and sustained processes in prospective memory and working memory. Cereb Cortex 19:1208-1221. CrossRef Medline

Rugg MD, Fletcher PC, Chua PM, Dolan RJ (1999) The role of the prefrontal cortex in recognition memory and memory for source: an fMRI study. Neuroimage 10:520-529. CrossRef Medline

Sauseng P, Griesmayr B, Freunberger R, Klimesch W (2010) Control mechanisms in working memory: a possible function of EEG theta oscillations. Neurosci Biobehav Rev 34:1015-1022. CrossRef Medline 
Scimeca JM, Badre D (2012) Striatal contributions to declarative memory retrieval. Neuron 75:380-392. CrossRef Medline

Siapas AG, Lubenov EV, Wilson MA (2005) Prefrontal phase locking to hippocampal theta oscillations. Neuron 46:141-151. CrossRef Medline

Simons JS, Spiers HJ (2003) Prefrontal and medial temporal lobe interactions in long-term memory. Nat Rev Neurosci 4:637-648. CrossRef Medline

Simons JS, Owen AM, Fletcher PC, Burgess PW (2005) Anterior prefrontal cortex and the recollection of contextual information. Neuropsychologia 43:1774-1783. CrossRef Medline

Spaniol J, Davidson PS, Kim AS, Han H, Moscovitch M, Grady CL (2009) Event-related fMRI studies of episodic encoding and retrieval: metaanalyses using activation likelihood estimation. Neuropsychologia 47: 1765-1779. CrossRef Medline
Tulving E (1985) Memory and consciousness. Can Psychol 26:1-12. CrossRef

van der Meer MA, Redish AD (2011) Theta phase precession in rat ventral striatum links place and reward information. J Neurosci 31:2843-2854. CrossRef Medline

Wimmer GE, Shohamy D (2012) Preference by association: how memory mechanisms in the hippocampus bias decisions. Science 338:270-273. CrossRef Medline

Yonelinas AP (2002) The nature of recollection and familiarity: a review of 30 years of research. J Mem Lang 46:441-517. CrossRef

Yonelinas AP, Jacoby LL (1995) The relation between remembering and knowing as bases for recognition: effects of size congruency. J Mem Lang 34:622-643. CrossRef 